\title{
Isolated Unilateral Hypoglossal Nerve Palsy: A Delayed Sequel of Covid-19
}

\author{
Ummer Karadan $^{1}$, Robin George Manappalli1 ${ }^{2}$, Mohan Leslie Noone ${ }^{1}$, Chellenton Jayakrishnan ${ }^{1}$, Ali Noufal ${ }^{3}$
}

Departments of ${ }^{1}$ Neurology, ${ }^{2}$ Internal Medicine and ${ }^{3}$ Physical Medicine and Rehabilitation, Baby Memorial Hospital, Calicut, Kerala, India.

\section{Corresponding Author:}

Dr Robin George Manappallil

Email: drrobingeorgemp1@gmail.com

This is an Open Access article distributed under the terms of the Creative Commons Attribution License (creativecommons.org/ licenses/by/3.0).

Received : February 7,2021

Accepted : April 13,2021

Published : May 25, 2021

\begin{abstract}
Background: The Corona virus disease (Covid-19) pandemic, caused by severe acute respiratory syndrome coronavirus 2 (SARS-CoV-2), is known to have several respiratory complications. However, some neurological complications have also been reported in critically ill patients with co-morbidities. Case Report: Our patient, a hypertensive, had mild flu like symptoms and was diagnosed to have Covid-19 infection. He was managed symptomatically and recovered. About 3 months later, he developed left hypoglossal nerve palsy. Conclusion: Isolated unilateral hypoglossal nerve palsy is a rare scenario, and its presentation as a delayed neurological sequel in Covid-19 infection, to the best of our knowledge, has not been reported before.
\end{abstract}

Keywords: COVID-19; Hypoglossal Nerve; Nervous System Diseases; Paralysis; SARS-CoV-2.

\section{Introduction}

The Corona virus disease (Covid-19), caused by severe acute respiratory syndrome coronavirus 2 (SARS-CoV-2), was first reported from Wuhan, China, in December 2019. Since then, the virus has spread rapidly globally; and the World Health Organization declared Covid-19 as a pandemic on March 11, 2020. Majority of Covid-19 patients have fever with mild to moderate respiratory symptoms. Though lungs are the most severely affected organ, neurological manifestations have also been observed in advanced stages of the disease. Isolated unilateral hypoglossal nerve palsy has multiple aetiologies. However, its presentation as a delayed sequel of Covid-19 has not been described yet.

\section{Case Report}

A 40-year-old male presented to the Medicine department with complaints of low-grade fever and dry cough for 2 days. He was a hypertensive, taking oral telmisartan (40 mg once daily). He was diagnosed to have Covid-19 infection (by RT-
PCR), and was managed symptomatically. About 3 months later, he presented to Neurology department with acute onset and gradually progressive dysarthria of 2 weeks duration. There were no other neurological symptoms. There was no history of trauma or recent vaccination. On examination, his vitals were stable. Neurologically, his tongue was deviated to the left side with wasting and decreased power on pushing the tongue against the cheek [Fig.1]. Fasciculations were present. Gag reflex and taste sensations were normal. Other systemic examinations were normal.

His blood investigations like complete blood counts, renal and liver parameters, electrolytes, calcium, magnesium, thyroid stimulating hormone and glycosylated haemoglobin were normal. Contrast enhanced magnetic resonance imaging of head and neck was normal. Epstein Barr virus IgM and $\mathrm{IgG}$ were non-reactive. Mantoux test and antinuclear antibodies were negative. Cerebrospinal fluid analysis was normal. Oropharyngeal fiber endoscopy did not show any abnormality. Electrocardiogram and chest X-ray were also 
normal. Oro-motor exercises for tongue along with range of motion and lingual strengthening exercises were started. Oral methyl-cobalamin (1500 mcg daily) and vitamin D (60000 units once weekly) were also given. On review after 3 weeks, he had showed improvement in speech and tongue atrophy. He was lost for further follow-up as he went off to the Gulf.

\section{Discussion}

SARS-CoV-2 was first isolated from the lower respiratory tract. They are a group of single stranded RNA viruses with genome lying within a helical nucleocapsid. The virus, after binding to the host cell receptor, fuses its envelope with the host cell membrane; and releases its nucleocapsid into the host cell cytoplasm. Later, the viral RNA multiplies and viral proteins are synthesized within the host cell. These viral proteins reassemble with the viral genome and form new virus particles, which in turn enter uninfected cells and the viral spread occurs. The SARS-CoV-2 virus utilizes the angiotensin-converting enzyme-2 (ACE-2) receptor for entering into the host cells. These receptors are well expressed on lungs and arterial and venous endothelial cells, and also in endothelial cells of cerebral capillaries. The virus reaches the brain by haematogenous spread and break the blood-brain barrier. It can also enter the brain trans-neuronally via the olfactory system, across the cribriform plate [1]. In the presence of comorbidities like diabetes and hypertension, these ACE-2 receptor expression get enhanced in the brain, causing neurotropism of the SARS-CoV-2 virus [2].

The common neurological symptoms are headache, myalgia and malaise. Other neurological manifestations include agitation and confusion, dizziness, diffuse corticospinal tract signs, seizures, taste and smell impairment, and skeletal muscle injury. The SARS-CoV-2 virus can affect both the central and peripheral nervous system. Neurological complications can occur either

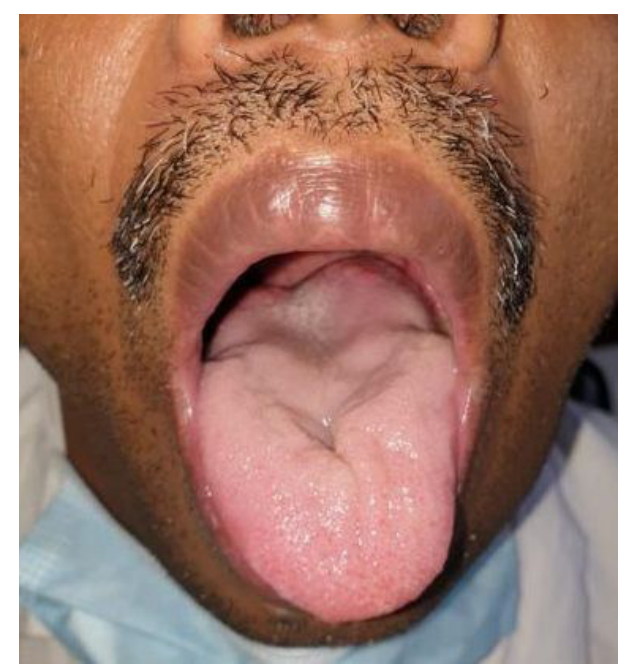

Fig.1: Left hypoglossal nerve palsy with tongue wasting.

because of direct viral invasion or immunological reaction or due to hypoxic metabolic changes. Some of the observed central nervous system complications include meningitis and encephalitis, acute hemorrhagic necrotizing encephalopathy, cerebral venous thrombosis, posterior reversible encephalopathy syndrome, acute disseminated encephalomyelitis, metabolic or hypoxic encephalopathy and stroke. The peripheral nervous system complications are isolated cranial nerve palsies, vision loss, neuralgic pain, Guillain-Barré syndrome, Miller Fisher syndrome, facial diplegia, myositis and rhabdomyolysis $[3,4]$.

Cranial nerve palsies are rare in Covid-19 infection. Oculomotor and abducens nerve palsies have been reported [5,6]. The probable pathogenesis is an inflammatory reaction against the SARS-CoV-2 virus and inflammation of vessels supplying the nerve trunk [6]. Hypoglossal nerve palsy is commonly seen in association with other cranial nerves abnormalities; however, its isolated unilateral presentation is rare. Some of the common causes of isolated unilateral hypoglossal nerve palsy are nasopharyngeal carcinomas, carcinomatous meningitis, skull base metastasis, trauma, dolichoectasia of the vertebral artery, extra-cranial internal carotid artery dissection, hypoglossal schwannoma, Epstein Barr virus 
infection, post-vaccination cranial neuritis, multiple myeloma, tubercular meningitis, chordoma, dural arteriovenous fistula, internal carotid artery aneurysm, rheumatoid arthritis, clival tumour, posterior fossa arachnoid cyst, acute disseminated encephalomyelitis and acute inflammatory demyelinating polyneuropathy.

Our patient had isolated left hypoglossal nerve palsy, which is a rare scenario. He was a hypertensive and had Covid-19 infection about 3 months ago. Cranial nerve palsies involving the oculomotor and abducens nerves have been observed with Covid-19 infection. With the regular causes of hypoglossal nerve palsy being ruled out, Covid-19 was assumed to be the probable aetiology in our patient. There has been a report of probable Covid-19 induced isolated unilateral hypoglossal nerve palsy [7]. However, our patient developed the palsy as a delayed sequel. To the best our knowledge, this is the first-time reporting of such a scenario.

\section{Conclusion}

Covid-19 infection can cause neurological complications affecting both central and peripheral nervous systems. Cranial nerve palsies involving oculomotor and abducens nerves have been observed with Covid-19 infection. It can cause cranial nerve palsies as a delayed sequel. However, isolated unilateral hypoglossal nerve palsy as a delayed sequel of Covid-19 has not been reported before.

Contributors: UK, MLN, CJ: Critical revision of manuscript and treating neurologist; RGM: concept and design of case report, reviewed the literature, manuscript preparation, and treating physician; AN: critical revision of manuscript and treating physiatrist. RGM will act as a study guarantor. All authors approved the final version of this manuscript and are responsible for all aspects of this study.

Funding: None; Competing interests: None stated.

\section{References}

1. Natoli S, Oliveira V, Calabresi P, Maia LF, Pisani A. Does SARS-Cov-2 invade the brain? Translational lessons from animal models. Eur J Neurol, 2020;27:1764-1773.

2. Puelles VG, Lütgehetmann $\mathrm{M}$, Lindenmeyer MT, Sperhake JP, Wong MN, Allweiss L, et al. Multiorgan and renal tropism of SARS-CoV-2. N Engl J Med. 2020;383(6):590-592.

3. Helms J, Kremer S, Merdji H, Clere-Jehl R, Schenck $\mathrm{M}$, Kummerlen $\mathrm{C}$, et al. Neurologic features in severe SARS-CoV-2 infection. N Engl J Med. 2020;382:22682270.

4. Mao L, Jin $\mathrm{H}$, Wang $\mathrm{M}, \mathrm{Hu} \mathrm{Y}$, Chen $\mathrm{S}, \mathrm{He} \mathrm{Q}$, et al. Neurologic manifestations of hospitalized patients with coronavirus disease 2019 in Wuhan, China. JAMA Neurol. 2020;77(6):683-690.

5. Wei H, Yin H, Huang M, Guo Z. The 2019 novel cornoavirus pneumonia with onset of oculomotor nerve palsy: A case study. J Neurol. 2020;267:1550-1553.

6. Falcone MM, Rong AJ, Salazar H, Redick DW, Falcone $\mathrm{S}$, Cavuoto KM. Acute abducens nerve palsy in a patient with the novel coronavirus disease (COVID-19). J AAPOS. 2020;24(4):216-217.

7. Costa Martins D, Branco Ribeiro S, Jesus Pereira I, Mestre S, Rios J. Unilateral hypoglossal nerve palsy as a Covid-19 sequel: a case report. Am J Phys Med Rehabil. 2020;99(12):1096-1098. 